\title{
Chinese painting elements in the application of modern menswear design
}

\author{
Meizhen Jiang ${ }^{1, a}$ \\ ${ }^{1}$ Jiangxi Institute of Fashion Technology, Jiangxi, Nanchang, 330201 \\ a107300691@qq,com
}

Keywords: Traditional Chinese painting. Background; Artistic conception; Emotional information; Ink painting

\begin{abstract}
Chinese painting is the fine tradition of the Chinese national culture, it has a long cultural history and rich heritage, occupies an important position in the world of art. With its unique artistic conception of Chinese painting and artistically, contains the rich thoughts in ancient China, daily life and emotional information of ancient cultural celebrities have passed. Traditional Chinese painting in the application of modern clothing also common occurance, ink painting design of fashion show are embodies the effect of cultural exchange. Brand clothing designers frequently to field trips to capture elements of different ethnic groups in the life.
\end{abstract}

\section{Introduction}

China has five thousand years of history of civilization, at the time of transformation and precipitation, has formed the attention of the world's unique characteristics of the Oriental artistic beauty, many artistic elements spread so far, with national characteristics such as: ink painting, painting, paper cutting, blue and white porcelain, facebook, Chinese knot, buckle, auspicious patterns, etc., these artistic elements has become the quintessence. National is the world, has the national characteristics of clothing has a calm temperament and profound artistic conception, in the modern rich culture breath it is always a beautiful scenery line, walk at the forefront of the world, leads the way forward. Many designers can get inspiration from Chinese traditional pattern, the embodiment of traditional Chinese painting is the spirit of Chinese national culture and great application in the clothing, for the application of traditional pattern is not only to innovate in the design, more of the culture of China in five thousand. We our excellent traditional culture can not be forgotten, after history of precipitation and accumulation history shows that it has been for sure. It is our choice in the design of a bright spot, make the finishing point of the pen, deduce another national fashion moments.

\section{The culture characteristics of Chinese painting and the formal beauty}

The culture characteristics of Chinese painting. Unity. Chinese painting and clothing in such aspects as style, color, design should be unified; Design and social connotation unified; Design form the unity of the diversity, etc. Decorative. Chinese painting is the whole dress later to the clothing decoration, traditional Chinese painting not only beautify, emphasize the effect but also can make up for the defects. Decorate themselves as human nature, as early as in ancient times people have used the leaves, animal bones sautoir, draw body feathers, shells and so on decoration of your body. To make up for the notes or weaken some characteristics of clothing modelling and structure, life is not everyone's body is a perfect, more or less have some shortcomings, such as leptin, fat body, inclined shoulder, shoulder, protruding abdomen, humpback, hold out a bosom, chicken breast, don't act as characteristics, such as through pattern can be designed according to the characteristics of the visual illusion of people can play a role of visual correction, make human body get perfect. Symbolic and implicated. Design as one of the decorative elements in people's life, it comes from life, reflect people's spiritual pursuit and yearning for a better life. In ancient Chinese painting, use specific symbolic objects as the pursuit of an image. Peony symbol with a silver spoon 
in her mouth; Ganoderma lucidum symbolize longevity; Symbol fruit harvest.

The aesthetic principles of Chinese painting. Pursuit of beauty is human nature, especially in the spiritual civilization and material civilization greatly improved today, people have more and more pursuit of beauty, for all the artistic creation, the use of law of formal beauty and the beauty of form performance are indispensable.

The beauty of symmetry and balance. Symmetry is also called it all together. According to the symmetrical Angle different, generally speaking, there are left and right sides is symmetrical, symmetrical, three types of inversion symmetry. Such as goalkeeper portrait is approximate symmetry in the folk art of China. Balance refers to the decorative elements such as abnormity equivalent, or with different quantity of way free configuration and psychological, on the vision of a stable and balanced form. It is not affected by the characteristics of the axis of symmetry or symmetric point limit, layout is free, but pay attention to the image center of gravity of the smooth, this kind of design has the outstanding theme, interspersed with freedom, image stretch beautiful, masculinity is flexible and athletic feeling strong, etc.

The beauty of the lines. Chinese painting lines is fruity and thick, have a sense of rhythm and don't feel stiff. Each must have the sense of flow and strength, give a person a kind of dish Qiu wolong feeling, feel the vitality of the extension expansion. The perfect combination of pen and ink as the main performance of Chinese painting language, is an indispensable content in traditional Chinese painting art, with the aid of all kinds of abstract of point, line, hard-wired, extract the bones of all things like to express the author's aesthetic emotion, this kind of point, line form of communication is the main Chinese painting abstract form.

\section{Innovation of Chinese painting in the clothing}

Constant innovation of Chinese painting, make clothing fashion elements added in the 21st century is a new concept. In the face of other international elements of innovation, as a modern people, in what way, what is the element contact, what kind of mentality in the face of the international fashion elements to go on, has become a with the mindset of continuous innovation rather than replicate mentality to adapt to the new challenges of the modern society.

Art is the need to constantly improve the innovation, to create a modern style of the size of Chinese painting, deeply understand the traditional Chinese painting type, shape, composition and color, will reappear in traditional Chinese painting of beautiful moral, strong interest in modern design, make the garment more connotation. If we just stand still copied those elements, so our excellent culture also become obsolete, what our predecessors left to also late after processing, it constantly through history take the essence to the dregs of the accumulation of precipitation come down. Innovation is not simply to carry the Chinese painting in the clothing, that will only make people feel far-fetched. Many designers break traditional clothing on creation idea of fixed pattern innovation, now some Chinese painting in the creation of concept is blended in among them. In clothing design can has the national characteristics of the classic elements, color and so on through the modern expression methods into their works, constitute a kind of ancient and modern fusion and contemporary popular artistic beauty.

There are a lot of Chinese painting in the clothing to create new ways. It can be through the body structure, dedicating the shape of the bones, can also according to the human body to the painting. Ink painting is flowing, can by ink performance, and then through the way of blowing the ink out natural form, the expression method is a kind of artist's style, but also to choose the right fabric. Natural texture is always at virtually produces aesthetic feeling, as if just like the nature of the created, for example of the waste paper; All kinds of leaves falling, etc. Can be coated with ink and paint on clothes. The artist's paintings use on the clothing with good can improve the flavor. Ethnic style of paper cutting, facebook, blue and white porcelain, etc can be creative application in the clothing. People's imagination is unlimited, as long as willing to bold innovation has the inspiration of mine forever.

\section{The application of traditional Chinese painting in the men's clothing design}

In the whole Chinese painting is a shining point in clothing, it can well reflect the designer's 
style. A different part of the design in the clothing as a result of the limitation of area and shape, the pattern of expression and appropriate, so it requires designers to choose suitable for the application form.

localization design application separately. The location in the clothing design according to the actual size to carry on the design of the specific areas of the clothing, in different place, collar, sleeves, front, bottom, back, shoulder, waist, hips, legs and other different parts to carry on the design, the application form in the most widely used in traditional costume. Typical traditional national costume in a work of men's clothing design on the application of the localization design. On the sleeves, using the form of painting according to the size of a reasonable layout. Around the bottom in the former, the use of plants and flowers and plants on the draw according to the size of the area of design.

Chinese painting in the animal and plant design. Traditional Chinese painting in the men's clothing design using the pattern of traditional Chinese painting, orchid, bamboo and birds and flowers, fruits pattern of ink painting, painting, lotus. Above these pattern uncluttered technique is used to reflect the characteristics of, also use the shading some technique to make the picture look more vivid, have rhythm feeling, hazy feeling, give a person a kind of natural, relaxed, ethereal feeling. Choose in fabrics on the natural cotton and linen fabrics and make the design more unique batik fabrics, national style. Men's wear both process and technology require strict and delicate. Men's clothing reaction to his taste, the correct dress to make them more confident, will also increase scores in her career. Men don't like the dress a lot, also don't like women's clothing color rich, design diversity.

The composition of Chinese painting and performance techniques. Men dressed in national wind clothing reflects the man's another kind of aesthetic feeling, temperament. Used in the design of the classic Chinese ink painting design, which USES the design of orchid, bamboo, lotus, painting of flowers and fruits. Orchid and bamboo is a flower of the four gentlemen, symbolism in ancient times to a certain extent, certainly in the modern does not lose its significance. "The silt but not imbrued, zhuo QingLian without demon", this is the character of lotus, flow love but not in the worldly temptation by secular. Painting of flowers and fruits pattern is also reflected in the graduation design, the fruit is a symbol of the fruitful, the significance of our work in a good harvest. Using the method of shading in the painting, make the picture looks empty solid, lifelike not too stiff. These patterns in the design and layout are drawn according to the style of clothing, different style, different parts of the area size, draw the corresponding design makes the picture look not too full don't seem so hollow. In the course of drawing, the different ways of composition. Using triangular composition, using rectangular composition, of course, will be in the draw drawing according to the corner of the clothing. In the clothing also applied the ink on the painting of "stay white" rich picture of the artistic conception, white space is an important expression of Chinese painting is a kind of language, white space in the clothing, the application of proper embodies the cultural taste of people. Art and design are interlinked, they can express to the designer's wishes. Ingenious designers can also use it for a good addition to the work, and realized the value of these patterns. In designer clothes work, use of high-end atmosphere can make the garment looks right grade, with bad will make the garment look at neither fish nor fowl, which requires the designer with a considerable amount of experience or experience.

The combination of traditional Chinese painting and fabrics. Choose the right fabrics make drawing up more convenient, natural cotton and linen and ink combination gives a person a kind of feeling of restoring ancient ways in China. Something restoring ancient ways is also one of the modern pop elements, by using the ancient elements express the people for years in a hurry, as time goes by a miss, also illustrates its existence value and the society. Popular reincarnation is verified this, all the rage now becomes obsolete, such as he again is a rebirth.

The combination of traditional Chinese painting and detail elements. In clothing design the details of the application of the Chinese tunic suit collar design, tang suit dish buckle design, the design of raglan sleeve. Chinese tunic suit has a strong political revolution and symbolic of ideology, then the Chinese tunic suit gave the impression is conservative, but in the combination of modern 
design of the lapel collar design makes it a more modern breath. Tang suit is one of the ancient Chinese clothing, including the iconic dish buckle as deck lives, one of the popular elements of personality has been used up to now, not only domestic designers use the button even abroad some designers also draw the essence of clever application. In the graduation design, buckle with elements of modern suits and plate combined with modern cultural characteristics. Button using cotton and linen fabrics and batik fabrics as button raw materials through reasonable collocation achieve harmony. Raglan sleeve design to make clothing more perfect, reflect gentleman gentleman, have a type, reflects the fashion sense in the whole series.

\section{Conclusion}

In short, in the process of design, combining the traditional Chinese painting and fabrics of a reasonable and orderly to play with the whole series. National wind of Chinese paintings menswear show man gentle, calm and steady, strong cultural connotation temperament, this is also the modern people lack of something. Chinese painting conveys positive energy and now we need to learn, Chinese painting can cultivate one's temperament, can make short-tempered person composed with connotation.

\section{Reference}

[1] imitations. Elements of Chinese painting in the application of custom clothing design [J] mass literature and art, 2014 (19).

[2] Linda; Cui Rong capacity. Chinese traditional elements in the men's products in the design of decoration application [J] of shandong textile economy, 2012 (02).

[3] Fifi. Design elements in the application of modern Chinese painting [J] art education, 2015 (9). 